\title{
Positronium Formation in Organic Liquids
}

\author{
A. Karbowski, K. Fedus, K. SŁużewski, J. Bruzdowska and G. Karwasz \\ Institute of Physics, Nicolaus Copernicus University, Grudziądzka 5, 87-100 Toruń, Poland
}

\begin{abstract}
This paper reports on the results of positron annihilation lifetime measurements of three organic liquids: benzene $\left(\mathrm{C}_{6} \mathrm{H}_{6}\right)$, cyclohexane $\left(\mathrm{C}_{6} \mathrm{H}_{12}\right)$, and methanol $\left(\mathrm{CH}_{3} \mathrm{OH}\right)$. The lifetime spectra are acquired at different temperatures for non-degassed, degassed, and oxygen-saturated samples, at temperatures between $5{ }^{\circ} \mathrm{C}$ and $25^{\circ} \mathrm{C}$. The spectra are analyzed using a standard three-exponential model. The influence of oxygen on each lifetime and intensity component is discussed.
\end{abstract}

DOI: $10.12693 /$ APhysPolA.132.1466

PACS/topics: 78.70.Bj, 82.30.Gg, 36.10.Dr

\section{Introduction}

Liquids were one of first targets for positronannihilation investigations. Extensive measurements showed rather long lifetimes, that indicated formation of ortho-positronium (o-Ps), with lifetimes $(2-3 \mathrm{~ns})$ much shorter than for the $o$-Ps in vacuum (142 ns). In porous glasses, the $o$-Ps lives up to tens of ns [1]. Positron lifetimes are much shorter, $100-200$ ps in metals [2] or semiconductors [3]. Tiny (few ps, imposed on 220 ps) differences of positron lifetimes in Czochralski-grown silicon allowed to detect clustering of oxygen atoms around defects $[4,5]$. In polymers, monitoring $o$-Ps lifetime (with similar values to those in liquids) became a powerful tool to study phase transitions [6] and dynamics of peristaltic vibrations of the chains [7]. A new generation of positron emission tomography (J-PET) [8] triggered again the interest in positron annihilation in organic molecules, and in particular in presence of oxygen, but expected differences between specific human tissues are tiny.

A new generation of positron lifetime spectrometers, with resolution down to $160-180$ ps [9] allowed to find matching between experiments in high-purity, monocrystal metals and theories [2]. Decomposition of lifetimes into very short (100-200 ps) and longer components (150-300 ps) allowed in metal to determine the kind and concentration of point-like defects; similarly in semiconductors [10]. Measurements with energy-tuned beams in gas phase allowed to discover low-energy laying resonances for a number of simple organic molecules, including hydrocarbons [11]; these resonances influence significantly the overall probability for positronium formation.

For liquids, the pioneer positrons measurements were done with rather poor time resolutions, so short lifetime components were not evidenced. Although the importance of $\mathrm{O}_{2}$ molecule in positron annihilation studies is a well-known fact, little quantitative data exist. There is practically no information on o-Ps lifetimes and intensities as a function of oxygen content at different physical conditions (temperature, presence of magnetic and electric fields). Some more quantitative studies on $o$-Ps paramagnetic quenching by $\mathrm{O}_{2}$ were undertaken in $[12,13]$. In the first paper [12] the influence of magnetic field on the ortho-para conversion induced by $\mathrm{O}_{2}$ in some plas- tic membranes is investigated, while more recent work deals with the role of oxygen solubility in different organic liquids. Excluding these works, most of experimental data on paramagnetic quenching come from relatively "old" papers (e.g. [14]) using positron annihilation lifetime (PAL) spectrometers with inferior time resolutions when compared with currently available systems. Consequently, the $o$-Ps lifetimes were usually derived through the two-component decomposition of acquired PAL spectra. Nowadays novel coincidence systems allow to resolve at least three different annihilation routes in organic liquids and solids. Some authors report even fourcomponent analysis $[15,16]$. Despite these trends, there are no quantitative data about $\mathrm{O}_{2}$ influence on the behaviour of other annihilation channels - different from the pick-off process of $o$-Ps.

Here, we report measurements of positron lifetimes in three organic liquids (benzene, cyclohexane and methanol) in the function of temperature and in a controlled atmosphere. The spectra are analyzed using standard three exponential model. The influence of paramagnetic quenching by $\mathrm{O}_{2}$ molecule on each lifetime and intensity component is discussed in detail. The goal of this work is to initiate more quantitative studies on positron annihilation with respect to the oxygen content in organic materials, and possibly human living tissues.

\section{Experimental setup}

The positron annihilation lifetime spectra (PALS) were measured using the fast-fast coincidence ORTEC PLS system equipped with plastic scintillators (St. Gobain BC418) and RCA 8850 photomultipliers [17]. The prompt time resolution of the system was $210 \mathrm{ps}$ in full width at half maximum (FWHM). The positron source ${ }^{22} \mathrm{Na}$ with activity $10 \mu \mathrm{Ci}$ was tightly sealed in $7 \mu \mathrm{m}$ thick envelope made of Kapton foil. The source was immersed in studied liquids that were placed inside a metal vacuum chamber pumped by Varian DS 42 Dual Stage Rotary Vane Pump. The temperature of the chamber was controlled by the Peltier cooler. Measurements were carried out as a function of temperature in three regimes: (i) in the presence of oxygen under normal pressure of the air, (ii) after degassing the chamber by a standard 
freeze-thaw technique (i.e. without oxygen), and (iii) after filling the space above liquid in the cell with oxygen under normal pressure (and room temperature). We assume that liquids were saturated with oxygen in all temperatures: the solubility of oxygen in the studied organic compounds changes little with temperature (and is pretty high, as compared to water) amounting as a mole fraction (in $10^{-3}$ units) at $10^{\circ} \mathrm{C}$ and $25^{\circ} \mathrm{C}$ to 1.245 and $1.245,0.788$ and $0.810,0.426$ and 0.419 for $\mathrm{C}_{6} \mathrm{H}_{12}$, $\mathrm{C}_{6} \mathrm{H}_{6}, \mathrm{C}_{3} \mathrm{OH}$, respectively [18]. Control measurements were done in oxygenated samples after evacuating oxygen (the same freeze-thaw). Typically, $4 \times 10^{5}$ total counts were accumulated in each measurement.

The analysis of lifetime spectra was realized with the LT package created by Kansy [19]. Three exponential components are traditionally ascribed to $p$-Ps decay $\left(\tau_{1}, I_{1}\right)$, annihilation of free positrons $\left(\tau_{2}, I_{2}\right)$, and $o$-Ps decay $\left(\tau_{3}, I_{3}\right)$ convoluted with the instrumental resolution curve were assumed. The source correction due to the positron absorption in the Kapton envelope was also applied $\left(I_{s}=6 \%\right.$ and $\left.\tau_{s}=380 \mathrm{ps}\right)$. The fits of three exponential model to PAL spectra were performed assuming a fixed lifetime value for $p$-Ps decay, $\tau_{1}=125 \mathrm{ps}$ that corresponds to its intrinsic lifetime in vacuum. Differently from our previous reporting [20] we did not fix the ratio between $o$-Ps and $p$-Ps intensities $3: 1$ (that is the value for Ps formation in vacuum, see experiment in Ref. [21]. An overall uncertainty on lifetimes and inten-
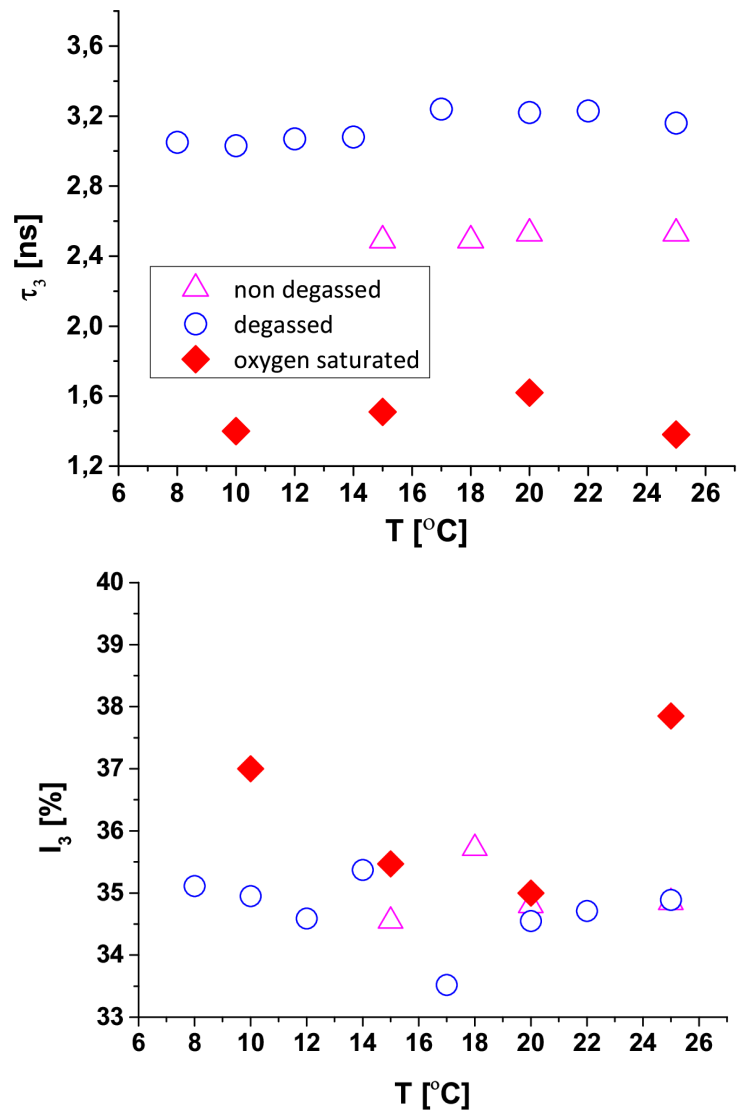

sities, due to experimental statistics, possible systematic errors and assumption on the analysis, is $10 \%$.

\section{Results}

Present results show that the analysis into three lifetimes gives high quality of fits for all cases. In degassed cyclohexane at $20^{\circ} \mathrm{C}$ the three lifetimes $\left(\tau_{3}, \tau_{2}, \tau_{1}\right)$

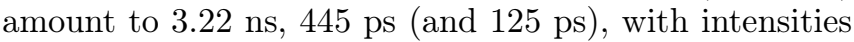
$35 \%, 44 \%$ and $21 \%$, respectively (and the variance 1.05 ). Generally, results for benzene (reported in detail previously [20]) and cyclohexane are quite similar; this probably reflects similar cross-sections in the gas phase [22].

An essential conclusion of present measurements is that the saturation with oxygen (or air) lowers significantly the $o$-Ps lifetime (by a factor of 2 approximately.) This was noticed already in pioneer experiments [14] and fully confirmed in more recent data from Milan Politecnics $[23,24]$. Our measurements show that in atmosphere of air the $\tau_{3}$ lifetime is in-between degassed and oxygensaturated samples, see Table I (for benzene) and Fig. 1 (for cyclohexane) and Fig. 2 (for methanol). Figure 1 and 2 show also that annihilation parameters change little with temperature. In benzene between $8{ }^{\circ} \mathrm{C}$ and $5^{\circ} \mathrm{C}$ we observed a drastic drop of $\tau_{3}$ (from 3.0 ns to $1.8 \mathrm{~ns}$ in degassed samples) that reflects the solidification of the liquid.
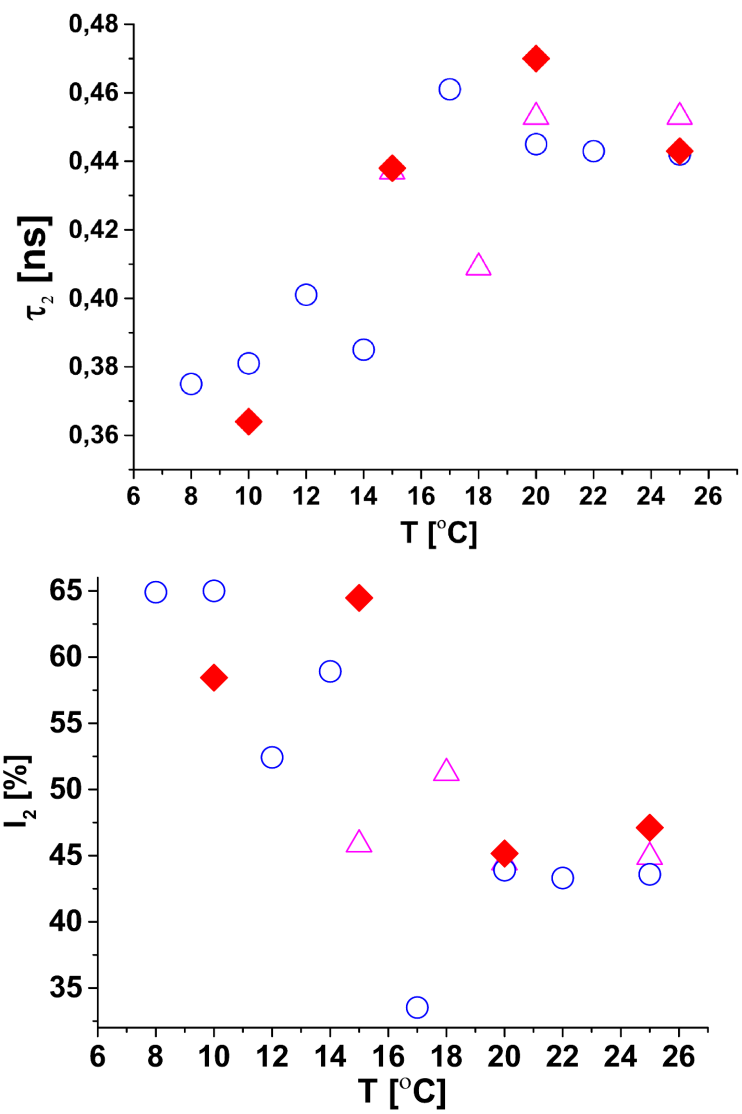

Fig. 1. Positron lifetimes $\tau_{3}, \tau_{2}$ and intensities $I_{3}, I_{2}$ as a function of temperature in non-degassed $(\Delta)$, degassed $(\downarrow)$ and oxygen-saturated $(\mathrm{O})$ cyclohexane $\left(\mathrm{C}_{6} \mathrm{H}_{12}\right)$. 

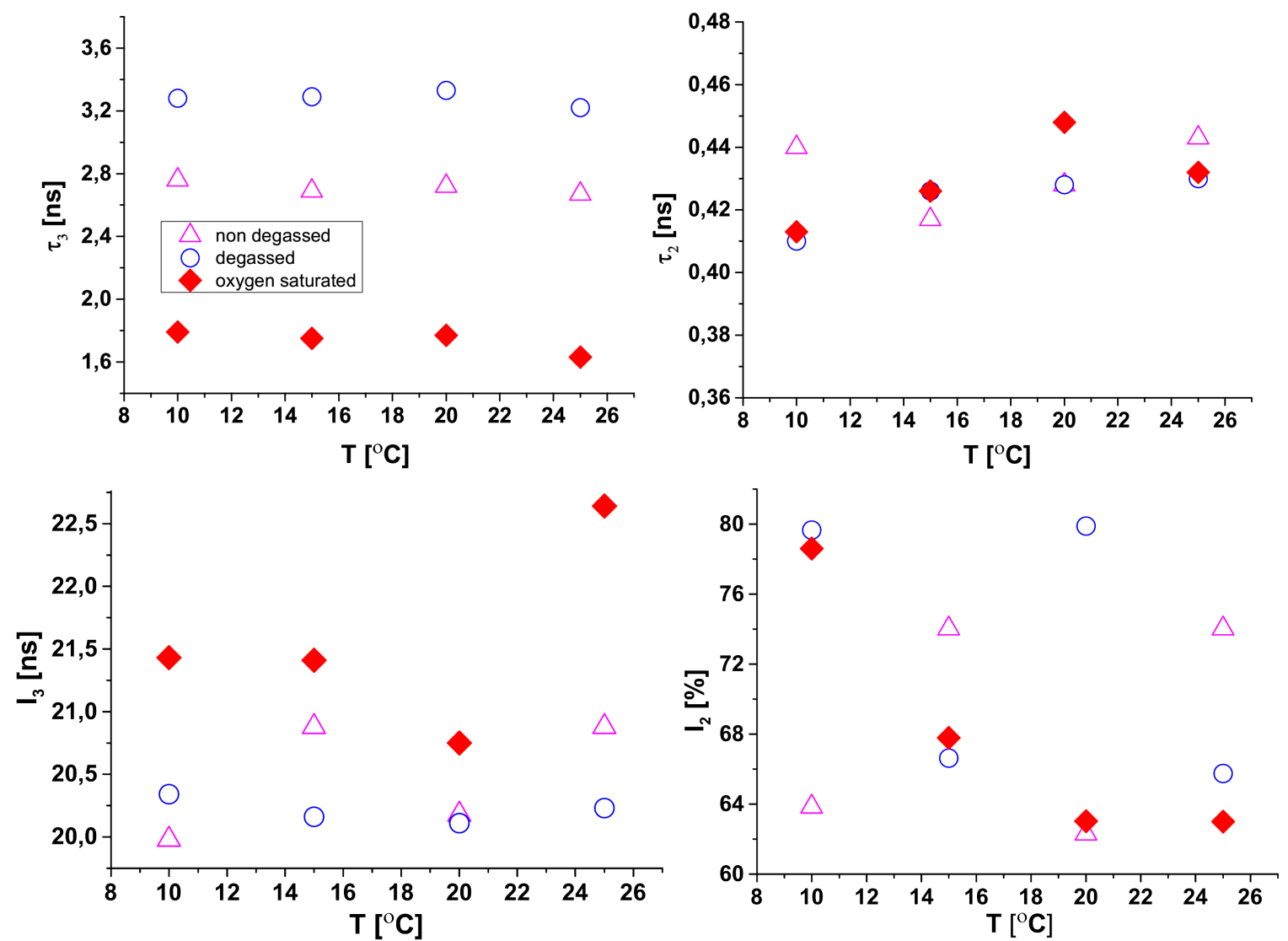

Fig. 2. As in Fig. 1 but for methanol $\left(\mathrm{CH}_{3} \mathrm{OH}\right)$.

TABLE I

Comparison positron lifetime analysis for benzene $\left(\mathrm{C}_{6} \mathrm{H}_{6}\right)$ at room temperature. In present analysis the $\tau_{1}$ was fixed.

\begin{tabular}{c|c|c|c|c|c|c|c|c}
\hline \hline$\tau_{1}$ & $I_{1}$ & $\tau_{2}$ & $I_{2}$ & $\tau_{3}$ & $I_{3}$ & $\tau_{4}$ & $I_{4}$ & \\
{$[\mathrm{~ns}]$} & {$[\%]$} & {$[\mathrm{ns}]$} & {$[\%]$} & {$[\mathrm{ns}]$} & {$[\%]$} & {$[\mathrm{ns}]$} & {$[\%]$} & Source \\
\hline 0.142 & 20.5 & 0.44 & 36.4 & 3.18 & 43.1 & - & - & {$[15]$, degas. } \\
- & 64.2 & - & - & 3.10 & 35.8 & - & - & {$[28]$, degas. } \\
0.148 & 18.1 & 0.416 & 37.4 & 3.26 & 39.2 & 1.18 & 5.3 & {$[23]$, degas. } \\
0.100 & 12.0 & 0.360 & 44.0 & 2.56 & 37.0 & 1.08 & 7.2 & {$[24]$, air satur. } \\
0.130 & 12.0 & 0.360 & 41.0 & 3.25 & 38.0 & 1.8 & 8.6 & {$[24]$, air degas. } \\
0.38 & 60.0 & - & - & 3.1 & 40.0 & - & - & {$[29]$} \\
- & 70 & - & - & 2.65 & 30 & - & - & {$[30]$} \\
- & 59 & - & - & 2.50 & 41 & - & - & {$[31]$} \\
- & - & - & - & 3.24 & - & - & - & {$[14]$, degas. } \\
- & - & - & - & 2.62 & - & - & - & {$[14]$, air satur. } \\
- & - & - & - & 1.75 & - & - & - & {$[14]$, O 2 satur. } \\
- & - & - & - & 2.60 & - & - & - & {$[25]$ in air $(?)$} \\
0.20 & 65.2 & - & - & 2.39 & 34.8 & - & - & {$[26]$ in air $(?)$} \\
- & - & - & - & 3.14 & - & - & - & {$[27]$} \\
0.125 & 26 & 0.483 & 36 & 3.18 & 38 & - & - & pres. degas. \\
0.125 & 26 & 0.503 & 36 & 2.57 & 38 & - & - & pres. air \\
0.125 & 20 & 0.478 & 40 & 1.66 & 40 & - & - & pres. O ${ }_{2}$ \\
& & & & &
\end{tabular}

Table I gives a detailed comparison between present and literature results in benzene (at room temparatures, as declared in available references). Present $o$-Ps lifetime in oxygen saturated sample $\left(\tau_{3}=1.66 \mathrm{~ns}\right)$ agrees well with the only experimental result, $1.75 \mathrm{~ns}$ [14], that we are aware of. Note that other parameters of the current three-term analysis remain practically unchanged by $\mathrm{O}_{2}$ within the present uncertainties. In particular presence of oxygen practically does not change the intensity of the third lifetime, being comprised in benzene between some $42-47 \%$ and some $37-41 \%$ in cyclohexane, see Fig. 1 for the latter. Presently derived lifetimes $\left(\tau_{2}=380-460 \mathrm{ps}\right.$ for all the three compounds) and intensities $I_{2}$ and $I_{1}$ at room temperature agree within uncertainties with three and four-component analysis of Consolati and co-workers [23, 24] and Mogensen [15]. All these data suggest that paramagnetic quenching has no influence either on the very $o$-Ps formation or on the other annihilation routes. A similar reduction of the $\tau_{3}$ in all three liquids presently studied suggest that the $\mathrm{O}_{2}$ induced quenching of $o$-Ps occurs at a very similar rate.

In all measurements the ration between $I_{3}$ and $I_{1}$ was found smaller than $3: 1$, quite close to $3 / 2: 1$, independent of presence of oxygen and the type of liquid. However, some indications appeared that the $I_{1}$ intensity gets reduced in low temperatures (i.e. in degassed cyclohexane at $12{ }^{\circ} \mathrm{C}$ the $I_{3}$ to $I_{1}$ ratio is $3: 1$ ). Alternatively, the analysis would require $\tau_{1}$ reduced below $125 \mathrm{ps}$, like it was observed in some measurements, see Table I.

Interesting differences occur in intensities, particularly for degassed samples, where the following relations were 
found at room temperature (i) for $I_{2}$ : $63 \%$ methanol $>44 \%$ cyclohexane $>36 \%$ benzene and (ii) $I_{3}: 41 \%$ benzene $>38 \%$ cyclohexane $>20 \%$ methanol. While $I_{3}$ in $\mathrm{C}_{6} \mathrm{H}_{6}$ and $\mathrm{C}_{6} \mathrm{H}_{12}$ are comparable, the $I_{3}$ intensity in $\mathrm{CH}_{3} \mathrm{OH}$ is much lower. In other words formation of positronium in methanol is much less probable (the summed $I_{1}$ and $I_{3}$ intensities amount to some $35 \%$ ), than in non-polar benzene and cyclohexane (55\% in the latter). The effect of the permanent dipole moment on reduced probability of positronium formation (i.e. the increased probability of the direct annihilation with the thermalized positron) requires further investigations (see also discussion in our previous paper [20]).

\section{Conclusions}

The most significant result of present measurements is the confirmation of positron quenching by the solvated oxygen. Remains surprising (even if known from the very pioneer measurements [28]) that values of the $o$-Ps lifetimes (in degassed and oxygen saturated samples) depend little on the chemical composition: measurements in compounds like dimethylpropane would be of interest for comparison. For a series of normal alkanes Gray et al. [28] observed also a slight dependence of $\tau_{3}$ on the length of the chain: re-analysis for $\tau_{2}$, and in partricular for $\tau_{1}$ would be of interest.

A more detailed quantitative study of quenching in function of oxygen content would be desirable, as the oxygen solubilities in cyclohexane and methanol differ by a factor of $3[18]$ but presently observed changes in $\tau_{3}$ are almost the same. Such studies would be extremely important in view of a new generation of positron emission tomography, see Ref. [8] that aim to distinguish not only the very annihilation event but also lifetimes of positrons in living tissues: it is well known that the oxygenation level is lower in tumours than in normal cells, see Ref. [20] for discussion. Little dependence of the annihilation rate on temperature in organic liquids would be a promising feature for such a PET.

On experimental side, re-analysis (and remeasurements) in view of more precise determination of the shortest lifetime ( $p$-Ps) value and its intensity is of interest. Finally, a contribution from theory in order to explain observed lifetimes would be extremely important.

\section{Acknowledgments}

This work is supported by the Grant 2014/15/D/ST2/02358 of National Science Center in Poland.

\section{References}

[1] J. Kullmann, D. Enke, S. Thraenert, R. KrauseRehberg, M. Beiner, Opt. Appl. XLII, 281 (2012).

[2] R.S. Brusa, W. Deng, G.P. Karwasz, A. Zecca, Nucl Instrum. Methods Phys. Res. B 194, 519 (2002).

[3] G.P. Karwasz, A. Zecca, R.S. Brusa, D. Pliszka, J. Alloys Comp. 382, 244 (2004).

[4] R.S. Brusa, W. Deng, G.P. Karwasz, A. Zecca, D. Pliszka, Appl. Phys. Lett. 79, 1492 (2001).
[5] G.P. Karwasz, R.S. Brusa, A. Misiuk, A. Zecca, Acta Phys. Pol. A 95, 575 (1999).

[6] A. Wieczorek, B. Zgardziniska, B. Jasińska, M. Gorgol, T. Bednarski, P. Białas, E. Czerwiński, A. Gajos, D. Kamińska, Ł. Kapłon, A. Kochanowski, G. Korcyl, P. Kowalski, T. Kozik, W. Krzemień, E. Kubicz, S. Niedźwiecki, M. Pałka, L. Raczyński, Z. Rudy, O. Rundel, N.G. Sharma, M. Silarski, A. Słomski, A. Strzelecki, W. Wiślicki, M. Zieliński, P. Moskal, Nukleonika 60, 777 (2016).

[7] P. Bandžuch, J. Krištiak, O. S̆auša, J. Zrubcová, Phys. Rev. B 61, 8784 (2000).

[8] P. Moskal, et al., Nucl. Instrum. Methods Phys. Res. A 764, 317 (2014).

[9] J. de Vries, A. Zecca, R.S. Brusa, S. Oss, Nucl. Instrum. Methods Phys. Res. A 275, 194 (1989).

[10] F. Tuomisto, in: Springer Handbook of Crystal Growth, Springer, Berlin 2010, p. 1551.

[11] G. Gribakin, J.A. Young, C. Surko, Rev. Mod. Phys. 82, 2257 (2010).

[12] G. Consolati, I. Genco, M. Pegoraro, L. Zanderighi, J. Polym. Sci. B Polym. Phys. 34, 357 (1996).

[13] B. Zgardzińska, W. Białko, B. Jasińska, Nukleonika 60, 801 (2015)

[14] J. Lee, G.J. Celitans, J. Chem. Phys. 42, 437 (1965).

[15] O.E. Mogensen, Positron Annihilation in Chemistry, Springer-Verlag, Heidelberg 1995.

[16] G. Consolati, F. Quasso, Appl. Phys. B 66, 371 (1998).

[17] A. Karbowski, J.D. Fidelus, G.P. Karwasz, Mater. Sci. Forum 666, 155 (2011).

[18] Solubility Data Series, Vol. 7, Oxygen and Ozone, Ed. R. Battino, Pergamon Press, Oxford 1981.

[19] J. Kansy, Nucl. Instrum. Methods Phys. Res. A 373, 235 (1996).

[20] A. Karbowski, K. Fedus, G. Karwasz, Acta Phys. Pol. B 48, 1593 (2017).

[21] T.S. Stein, W.E. Kauppila, C.K. Kwan, S.P. Parikh, S. Zhou, Hyperfine Interact. 73, 53 (1992).

[22] G.P. Karwasz, D. Pliszka, R.S. Brusa, C. Perazzolli, Acta Phys. Pol. A 107, 666 (2005).

[23] G. Consolati, D. Gerola, F. Quasso, J. Phys. Condens. Matter 3, 7739 (1991).

[24] A. Bisi, G. Consolati, N. Gambara, L. Zappa, Nuovo Cimento D 13, 393 (1991).

[25] S. Berko, A.J. Zuchelli, Phys. Rev. 102, 724 (1956).

[26] G.R.A. de Blonde, Ph.D. Thesis, University of Manitoba, 1972.

[27] B.J. Brown, Aust. J. Chem. 27, 1125 (1974).

[28] P.R. Gray, C.F. Cook, G.P. Sturm, J. Chem. Phys. 48, 1145 (1968).

[29] J.A. Merrigan, J.H. Green, S.J. Tao, in: Physical Methods of Chemistry, Ed. A. Weissberger, Vol. 1, Wiley, New York 1972, Pt. IID.

[30] K.P. Singh, R.M. Singru, C.N. Rao, J. Phys. Atom. Mol. Phys. 4, 261 (1971).

[31] C.R. Hatcher, T.W. Falconer, W.E. Millett, J. Chem. Phys. 32, 28 (1960). 